\title{
Smoking status and cause-specific discontinuation of tumour necrosis factor inhibitors in axial spondyloarthritis
}

Sizheng Steven Zhao ${ }^{1,2,3}$, Kazuki Yoshida, ${ }^{3,4}$, Gareth T. Jones ${ }^{5,6}$, David M. Hughes ${ }^{7}$, Stephen J. Duffield', Sara K. Tedeschi ${ }^{3}$, Houchen Lyu ${ }^{8,9}$, Robert J. Moots ${ }^{1,2}$, Daniel H. Solomon ${ }^{3,10}$ and Nicola J. Goodson ${ }^{1,2^{*}}$ (D)

\begin{abstract}
Background: The impact of smoking on TNF inhibition (TNFi) therapy is unclear. We examined the effect of smoking on all-cause and cause-specific TNFi discontinuation in axial spondyloarthritis (axSpA).

Methods: We used longitudinal data from the British Society for Rheumatology Biologics Register for Ankylosing Spondylitis (BSRBR-AS). Patients fulfilling the ASAS criteria for axSpA, who started their first TNFi, were eligible for analysis. Inverse-probability weights were used to balance differences in baseline disease severity and other confounders. We used marginal structural Cox proportional hazard models to estimate hazard ratios (HR) for TNFi discontinuation according to smoking status. In analyses of cause-specific discontinuation, competing risk events were considered as censoring, using inverse-probability weights.

Results: A total of 758 participants were included in the analysis (66\% male, mean age 45 years), providing 954 patient-years of follow-up. TNFi was discontinued in 174 (23\%) patients, among whom 26\% stopped due to infections, 20\% due to other adverse events and $44 \%$ due to inefficacy or other reasons. Thirty-four percent were current smokers and 30\% ex-smokers. Compared to never smokers, current smokers' risk of TNFi discontinuation was HR $0.79(95 \% \mathrm{Cl} 0.53$ to 1.20) and ex-smokers HR 0.68 ( $95 \% \mathrm{Cl} 0.45$ to 1.04). Our data did not show evidence that current smoking influenced discontinuation due to infections (HR $0.79,95 \% \mathrm{Cl} 0.40$ to 1.54 ), other adverse events ( $\mathrm{HR} 0.86,95 \% \mathrm{Cl} 0.41$ to 1.78 ) or inefficacy/other causes ( $\mathrm{HR} 1.44,95 \% \mathrm{Cl} 0.86$ to 2.41 ).
\end{abstract}

Conclusion: Baseline smoking status did not impact TNFi discontinuation in this UK cohort of axSpA participants.

Keywords: Axial spondyloarthritis, Ankylosing spondylitis, TNF inhibitor, Biologic DMARDs, Persistence, Effectiveness, Response, Discontinuation, Marginal structural model

\section{Background}

The aetiological and prognostic roles of cigarette smoking in rheumatoid arthritis are well-established, but its role in axial spondyloarthritis (axSpA) remains unclear. Clinically meaningful differences in response to TNF inhibitors (TNFi) have not been demonstrated in axSpA when continuous outcomes are used, such as the Bath Ankylosing Spondylitis Disease Activity Index (BASDAI) [1-3]. In contrast, analyses using binary response variables, such as

\footnotetext{
* Correspondence: ngoodson@liverpool.ac.uk

'Musculoskeletal Biology I, Institute of Ageing and Chronic Disease,

University of Liverpool, Liverpool, UK

${ }^{2}$ Department of Academic Rheumatology, Aintree University Hospital, Liverpool, UK

Full list of author information is available at the end of the article
}

BASDAI50 (50\% reduction), found current smokers to have significantly lower odds of TNFi response compared to never smokers $[1,4]$. Since all causes of discontinuation are often counted as non-response, increased drug discontinuation in smokers may explain this discrepancy.

One prior study reporting increased risk of treatment discontinuation in smokers did not adjust for socioeconomic status, comorbidities or baseline disease severity [4]. Since these are potential confounders or known predictors of TNFi discontinuation [5], limited conclusions can be drawn about the independent relationship between smoking and TNFi discontinuation.

Patients stop TNFi for several reasons, including inefficacy, adverse events or other reasons, such as patients'

(C) The Author(s). 2019 Open Access This article is distributed under the terms of the Creative Commons Attribution 4.0 International License (http://creativecommons.org/licenses/by/4.0/), which permits unrestricted use, distribution, and 
social circumstances. Infections are adverse events of special interest since smoking is a substantial risk factor for bacterial and viral infections [6]. Whether the effect of smoking differs according to each cause of TNFi discontinuation is not known. Once treatment is stopped for one reason (e.g. adverse events), the individual can no longer be at risk of other causes of discontinuation (e.g. inefficacy) in that treatment episode. When an event either precludes observation of the outcome of interest or modifies its probability, "competing risks" is present [7]. When competing risks are not rare, i.e. $>5 \%$ of the population, traditional survival analyses should not be used and additional methodological considerations are needed to appropriately understand a potential association between smoking and TNFi discontinuation.

The aim of the current analyses was to examine the impact of smoking on all-cause and cause-specific TNFi discontinuation in patients with axSpA, using marginal structural models to address the above methodological issues.

\section{Methods}

\section{Study design and population}

We used data from the British Society for Rheumatology Biologics Register for Ankylosing Spondylitis (BSRBRAS), a UK-wide prospective cohort study of patients fulfilling the Assessment of SpondyloArthritis international Society (ASAS) criteria for axSpA [8]. The current analysis focused on new users of TNFi (Humira, Enbrel/ Benepali, Cimzia and Simponi) from December 2012 to June 2017. Patients were assessed at baseline, 3, 6 and 12 months and annually thereafter, with additional follow-ups permitted in the interim. TNFi start and stop dates were ascertained from their medical notes. Participants who completed a valid baseline questionnaire (within 1 year before and 7 days after starting TNFi) and recorded smoking status were eligible for analysis.

\section{Exposure and outcomes}

Self-reported smoking status at baseline (current, ex- or never) was used to define the exposure. The outcomes of interest were time to all-cause and cause-specific TNFi discontinuation. Reasons for stopping were categorised in the BSRBR-AS as adverse events, inefficacy, symptom in remission, or other, with accompanying free-text to further elaborate. Each entry was manually reviewed and, where necessary, re-categorised. To allow comparison with existing studies, and so that each specific cause has sufficient events with which to perform the analyses, we grouped causes as (1) infection-related adverse events, (2) all other adverse events, and (3) inefficacy or other reasons (e.g. patient choice and social circumstances). The censoring date was defined as the last study visit or, if no data existed past the baseline visit, as 3 months (the minimum per protocol follow-up interval).

\section{Covariates}

The following covariates were recorded at baseline and chosen a priori for their possible associations with TNFi discontinuation based on prior literature [5]: age, gender, symptom duration, education, elevated baseline CRP (above upper normal limit), classification as ankylosing spondylitis (modified New York criteria), social deprivation (quintiles) [9], alcohol use (current, previous, never), comorbidity (categorised as 0,1 or $\geq 2$ from a list of 13 conditions [8]), body mass index (BMI), TNFi agent and year of TNFi initiation. Patient-reported variables were measured at each follow-up; those known to be associated with TNFi discontinuation include BASDAI, spinal pain, functional impairment (BASFI) and fatigue (Chalder Fatigue Scale). Since these variables capture more than just disease activity, we refer to them collectively as "disease severity" throughout the text.

\section{Statistical analysis}

Baseline participant characteristics were summarised by smoking status. Unadjusted comparison of time to allcause discontinuation according to smoking status was made using Kaplan-Meier estimators.

Prior studies considered each cause of TNFi discontinuation as censoring [10]. In traditional survival analysis, observation time for each subject is censored when they leave the study or at the end of follow-up. Furthermore, a key assumption of Cox models is that censored patients should be representative of remaining individuals at that time point (i.e., censoring is random). Patients who discontinue treatment are not representative of those who continue; therefore, Cox models cannot be used.

Marginal structural models use inverse-probability weights to account for confounders rather than adjusting for them in conventional outcome regression [11]. This approach is more flexible, allowing us to account for baseline imbalance in characteristics between smoking status, time-varying disease activity, and competing risk events within one model. For all following analyses, differences in baseline covariates between smoking categories were balanced using inverse probability of "treatment" weights (IPTW) [11] to allow unconfounded descriptive comparisons [12]. Causal inference is not straightforward regarding the effect of baseline smoking status on TNFi discontinuation, since we cannot randomly assign an individual to "having smoked for 20 years" at the onset of a hypothetical trial. IPTW provides an approach to estimate causal effects under theoretical ideal conditions and may be advantageous over 
regression adjustment (see Additional file 1). We derived IPTW using predicted values from a multinomial logistic regression model, using baseline smoking status as the dependent variable and all baseline covariates specified above as independent variables.

We applied baseline time-invariant inverse probability of censoring weights (IPCW) to account for participants excluded from the sample eligible for analysis, such that baseline characteristics of the analysis set resembled the eligible TNFi exposed cohort. These IPCWs were constructed in the same manner as IPTWs, with inclusion/ exclusion status as the dependent variable.

We used marginal structural Cox proportional hazards models to estimate hazard ratios of TNFi discontinuation according to baseline smoking status [11, 13]. Parameters were estimated using weighted pooled logistic models that are equivalent to Cox models when using discrete time [14] and allow the use of subject-specific time-varying weights. Time was split into integer months [13].

Censoring becomes non-random when competing risk events are specified as censoring. We therefore calculated time-varying IPCWs, such that censoring becomes random at each time point with respect to baseline characteristics and history of disease activity [15]. For analysis of each specific cause of TNFi discontinuation (e.g. infection), the other competing risk events (e.g. other reasons) were treated as censoring events.

Weighted pooled logistic models included only the outcome, exposure (smoking status) and time, which was included in all models as restricted cubic splines [13]. All weights were "stabilised" to have a mean of 1 , allowing the overall sample size to remain unchanged [16]. Further details of the methods are given in [13]. Multiple imputation was used for missing covariates. Analyses were conducted using Stata version 13.

\section{Results}

Among a total of 2420 participants in the BSRBR-AS, 840 commenced their first TNFi within the study period and had smoking status recorded at baseline. Eighty-two participants were excluded because they did not have a valid baseline assessment. Excluded participants had longer symptom duration, more frequently met classification criteria for AS and were more frequently male (full comparison in Additional file 1: Table S1). Seven hundred fifty-eight participants were included in the analysis set, providing 954 patient-years of follow-up. The median follow-up time was 1.0 year (inter-quartile range 0.4 to 2.0 years).

TNFi was discontinued in 174 (23\%) patients. The median time to discontinuation was 6 months (inter-quartile range 3 to 11). Among those who stopped TNFi, $26 \%$ stopped due to infections, $20 \%$ due to other adverse events and $44 \%$ due to inefficacy or other reasons, with no differences according to smoking status. Recoding of discontinuation causes is shown in Additional file 1: Table S2.

Baseline characteristics of the analysis cohort are shown in Table 1. Thirty-four percent of patients were current smokers, 30\% ex-smokers and 36\% never smokers. Current smokers were younger, were more frequently male and showed trends for having higher deprivation and lower educational attainment. Current smokers also more frequently had elevated CRP. The three groups did not differ in age of symptom onset. Current smokers reported worse baseline disease activity and functional impairment. Covariate imbalance was negligible (standardised mean differences <0.1) after weighting for IPTW (Additional file 1: Figure S1).

\section{All-cause TNFi discontinuation}

Using marginal structural models, hazard ratios for allcause TNFi discontinuation did not significantly differ in current (HR $0.79 ; 95 \% \mathrm{CI} 0.53$ to 1.20 ) or ex-smokers (HR $0.68 ; 95 \% \mathrm{CI} 0.45$ to 1.04 ), compared to never smokers. Unadjusted Kaplan-Meier estimators are shown in Additional file 1: Figure S2.

\section{Cause-specific TNFi discontinuation}

Hazard ratios for each of the three causes of TNFi discontinuation according to smoking status are shown in Table 2. Our data did not provide evidence that current or previous smoking affected discontinuation due to infection, other adverse events or inefficacy/other reasons.

\section{Discussion}

In this large UK cohort of axSpA participants, smoking was associated with significantly worse disease severity at initiation of TNFi therapy. However, TNFi discontinuation was not associated with baseline smoking status. Our results also did not provide evidence that smoking affected discontinuation due to infection-related or other adverse events. The results did suggest that current smokers may have higher risk of discontinuation due to inefficacy or other reasons.

The main strengths of this study are the quality of data and use of rigorous analyses. The BSRBR-AS cohort is representative of UK clinical practice, and its rich dataset allowed us to adjust for a large number of confounders. The use of inverse-probability weights allowed us to conduct improved time-to-event analysis for each cause of TNFi discontinuation (competing risk events), while accounting for baseline differences between exposure groups and time-varying disease activity within one model.

Our data did have some important limitations. While smoking has been recorded in detail, information on 
Table 1 Baseline characteristics and causes of discontinuation among 758 participants, according to smoking status

\begin{tabular}{|c|c|c|c|c|c|}
\hline & & $\begin{array}{l}\text { Never smoker } \\
(n=224)\end{array}$ & $\begin{array}{l}\text { Ex-smoker } \\
(n=177)\end{array}$ & $\begin{array}{l}\text { Current smoker } \\
(n=197)\end{array}$ & $P$ value \\
\hline \multicolumn{2}{|l|}{ Age, mean (SD) years } & $43.3(14.5)$ & $50.0(12.8)$ & $42.2(11.8)$ & $<0.001$ \\
\hline \multicolumn{2}{|l|}{ Male } & $167(62 \%)$ & $149(65 \%)$ & $188(73 \%)$ & 0.012 \\
\hline \multicolumn{2}{|l|}{ Meets mNY criteria for AS } & $164(61 \%)$ & $154(67 \%)$ & $162(63 \%)$ & 0.360 \\
\hline \multicolumn{2}{|l|}{ HLA-B27 positive ${ }^{+}$} & $143(70 \%)$ & $125(76 \%)$ & $148(80 \%)$ & 0.088 \\
\hline \multicolumn{2}{|l|}{ Elevated CRP* } & $150(57 \%)$ & $129(59 \%)$ & $161(67 \%)$ & 0.043 \\
\hline \multicolumn{2}{|l|}{ Age at symptom onset, median (IQR) years } & 26.0 (20.0 to 33.0) & 26.0 (22.0 to 34.0$)$ & 26.0 (21.0 to 33.0$)$ & 0.350 \\
\hline \multicolumn{2}{|l|}{ Symptom duration, median (IQR) years } & $12.4(4.2$ to 26.0$)$ & 20.7 (10.3 to 32.5) & 12.3 (5.5 to 22.9) & $<0.001$ \\
\hline \multicolumn{2}{|l|}{ BMI, mean (SD) } & $27.9(6.2)$ & $28.8(5.1)$ & $27.4(5.8)$ & 0.060 \\
\hline \multirow[t]{5}{*}{ Quintiles of Index of Multiple Deprivation } & 1, most deprived & $39(14 \%)$ & $33(14 \%)$ & $85(33 \%)$ & \multirow[t]{5}{*}{$<0.001^{* *}$} \\
\hline & 2 & $54(20 \%)$ & $30(13 \%)$ & $43(17 \%)$ & \\
\hline & 3 & $52(19 \%)$ & $53(23 \%)$ & $51(20 \%)$ & \\
\hline & 4 & $68(25 \%)$ & $56(24 \%)$ & $47(18 \%)$ & \\
\hline & 5 , most affluent & $58(21 \%)$ & $59(26 \%)$ & $30(12 \%)$ & \\
\hline \multirow[t]{5}{*}{ Highest level of education } & Secondary school & $72(27 \%)$ & 75 (33\%) & $114(45 \%)$ & \multirow[t]{5}{*}{$<0.001$} \\
\hline & Apprenticeship & $18(7 \%)$ & $24(10 \%)$ & $29(12 \%)$ & \\
\hline & Further education college & $83(31 \%)$ & $81(35 \%)$ & $71(28 \%)$ & \\
\hline & University degree & $70(26 \%)$ & $38(17 \%)$ & $29(12 \%)$ & \\
\hline & Further degree & $26(10 \%)$ & $11(5 \%)$ & $9(4 \%)$ & \\
\hline \multirow[t]{3}{*}{ Alcohol status } & Never & $31(11 \%)$ & $14(6 \%)$ & $30(12 \%)$ & \multirow[t]{3}{*}{$<0.001$} \\
\hline & Ex & $34(13 \%)$ & $43(19 \%)$ & $68(27 \%)$ & \\
\hline & Current & $205(76 \%)$ & $174(75 \%)$ & $158(62 \%)$ & \\
\hline \multirow[t]{3}{*}{ Number of comorbidities } & 0 & $163(61 \%)$ & 117 (51\%) & $137(54 \%)$ & \multirow[t]{3}{*}{$0.051^{* *}$} \\
\hline & 1 & 77 (29\%) & $68(30 \%)$ & $78(30 \%)$ & \\
\hline & $\geq 2$ & $28(10 \%)$ & $43(19 \%)$ & $41(16 \%)$ & \\
\hline \multicolumn{2}{|l|}{ BASDAI, median (IQR) } & 6.3 (4.8 to 7.4$)$ & $6.6(5.2$ to 7.9$)$ & 7.1 (5.5 to 7.9 ) & 0.001 \\
\hline \multicolumn{2}{|l|}{ Spinal pain, median (IQR) } & 6.0 (4.0 to 8.0$)$ & 7.0 (5.0 to 8.0$)$ & 7.0 (5.0 to 8.0$)$ & 0.002 \\
\hline \multicolumn{2}{|l|}{ BASFI, median (IQR) } & 5.7 (3.7 to 7.6$)$ & 6.6 (4.8 to 8.3$)$ & 7.0 (5.1 to 8.6) & $<0.001$ \\
\hline \multicolumn{2}{|l|}{ Chalder Fatigue Scale, median (IQR) } & 17.0 (14.0 to 21.0$)$ & 17.0 (13.0 to 21.0$)$ & 18.0 (13.0 to 22.0$)$ & 0.340 \\
\hline \multicolumn{2}{|l|}{ Remained on treatment } & $62(23 \%)$ & 49 (21\%) & $63(25 \%)$ & 0.670 \\
\hline \multirow[t]{3}{*}{ Stopped treatment } & Infection & $20(32 \%)$ & $18(37 \%)$ & $15(24 \%)$ & \multirow[t]{3}{*}{0.176} \\
\hline & Other adverse events & $17(27 \%)$ & $15(31 \%)$ & $13(21 \%)$ & \\
\hline & Inefficacy or other reasons & $25(40 \%)$ & $16(33 \%)$ & $33(56 \%)$ & \\
\hline
\end{tabular}

Data presented as mean (standard deviation), median (interquartile range), number (percentage). Comparisons used $t$-test for continuous variables, chi-square test for categorical variables. Italicized text highlights significant differences. Examples of "other reasons" included patient choice and social circumstances

${ }^{+}$HLA-B27 status available for 448 participants

*Above upper normal limit

**Non-parametric test for trend across ordered groups

$S D$ standard deviation, IQR interquartile range, $m N Y$ modified New York criteria for Ankylosing Spondylitis, BMI body mass index, BASDAI Bath AS Disease Activity Index, BASFI Bath AS Functional Index

time since smoking cessation was not recorded. The proportion stopping due to adverse events was higher, while inefficacy was much lower, than reported elsewhere; nevertheless, the proportion of TNFi discontinuation was in keeping with existing literature [10]. In clinical practice, there is often an overlap between how inefficacy and adverse events are defined and reported. For instance, inflammation in previously unaffected peripheral joints may be reported as an adverse event, when it would more appropriately reflect lack of disease control and efficacy; therapy is more likely to continue in the face of mild adverse events if they are highly effective for symptom control. Before 2016-that is, the majority of this study period-the UK National Institute for Health and Care Excellence stipulated that patients who did not demonstrate initial or maintained response 
Table 2 All-cause and cause-specific treatment discontinuation according to baseline smoking status, after balancing differences in baseline covariates and accounting for those excluded from the analysis

\begin{tabular}{llll}
\hline & Never smoker & Ex-smoker & Current smoker \\
\hline All-cause & Reference & 0.68 (0.45 to 1.04) & 0.79 (0.53 to 1.20) \\
Infection-related adverse events & Reference & 1.03 (0.55 to 1.96) & 0.79 (0.40 to 1.54) \\
Other adverse events & Reference & 1.02 (0.50 to 2.07) & 0.86 (0.41 to 1.78) \\
Inefficacy and other reasons & Reference & 0.73 (0.39 to 1.37) & 1.44 (0.86 to 2.41) \\
\hline
\end{tabular}

Results shown as hazard ratios (95\% confidence interval). Examples of "other reasons" included patient choice and social circumstances

to treatment would not be funded to use a second TNFi $[17,18]$. However, switching to a second TNFi was allowed if discontinuation was due to the development of a treatment-related adverse event. This may have influenced labelling of discontinuation as adverse events or other reasons. These errors would not affect analysis of all-cause discontinuation or discontinuation due to infection-related adverse events. It would have been interesting to examine the effect of changing smoking status at the individual level, but the number of such participants was too small to permit analysis. It is possible that TNFi stop dates in medical notes may be influenced by recall error; however, there is no evidence to suggest that such error differs according to smoking status, so we do not believe this will have introduced a bias. Lastly, the BSRBR-AS did not record pack-years to examine the effect of cumulative exposure. A significant effect is unlikely since none was found for ever vs never smokers.

There has been only one previous study of TNFi discontinuation and smoking in ankylosing spondylitis. Glintborg et al. reported increased risk of all-cause TNFi discontinuation in current and ex-smokers [4]. Compared to their study, our follow-up period was short. It is possible that differences in treatment persistence only becomes apparent with longer follow-up, although separation in Kaplan-Meier curves was clear by 2 years in their study [4]. Importantly, Glintborg et al. did not adjust for baseline differences in disease severity and BMI from their primary analysis, on account of the fact that these variables are potential mediators. Without adjusting for these known predictors of TNFi persistence $[5,19]$, it is difficult to attribute a causal effect of smoking on TNFi discontinuation. In DANBIO studies of psoriatic arthritis, significant differences in TNFi persistence were reported when analyses did not adjust for BMI and baseline disease severity [10], while analysis using essentially the same cohort but additionally adjusting for these covariates did not find such difference [20] (see Additional file 1: Table S4 for comparison).

Our negative results may have other explanations. The prevalence of current smoking was higher in DANBIO (43\%) than in the BSRBR-AS (29\%). Furthermore, we did not find smoking to be associated with age of symptom onset as previously reported [21]. It is possible that there are differences between the study cohorts. Our results are consistent with a growing body of evidence that smoking status does not independently impact TNFi treatment. In a study of obesity and TNFi response in axSpA, current smoking (as a covariate in their multivariable Cox regression model) was not associated with TNFi discontinuation (HR 0.92; 95\%CI 0.66 to 1.28) [19]. Smoking status at TNFi initiation also did not affect treatment response in several studies [2, 19, 22-25], including a recent analysis of the BSRBR-AS [3]. These results should persuade clinicians to dispel any subconscious bias that smokers may not benefit as much from TNFi treatment.

We cannot exclude the possibility that smoking in early stages of the disease causes more severe disease observed at TNFi initiation. It is unknown whether smoking cessation improves disease severity irrespective of treatment. Smoking cessation remains a priority since patients with rheumatic diseases have high burdens of cardiovascular disease and the combined impact of TNFi and smoking on malignancy risk is unknown. Furthermore, smoking may be associated with greater radiographic progression [26].

\section{Conclusion}

Smokers commencing TNFi were no more likely to discontinue treatment than never smokers in this large UK cohort of axSpA participants. To better estimate causal effects of smoking exposure, future studies would benefit from recording more detailed smoking history, and exploring cause-specific treatment discontinuation in larger cohorts with clearer recording of reasons for discontinuation.

\section{Additional file}

\footnotetext{
Additional file 1: Table S1. Characteristics of the 840 patients exposed to TNFi and had smoking status, according to whether they were eligible for longitudinal analysis or were excluded. Table S2. Recoding mis-labelled discontinuation causes in the registry data. Table S3. Descriptions of stabilised inverse-probability weights used in analyses. Table S4. Comparing results from studies of TNFi persistence according to covariates used in their analysis models, particularly whether they have accounted for baseline disease severity. Figure S1. Standardised mean differences (SMD) for baseline variables before and after inverse-propensity weighting. Figure S2. Kaplan-Meier curves comparing all-cause discontinuation between smoking status. (DOCX $141 \mathrm{~kb}$ )
} 


\section{Acknowledgements}

We are grateful to Professor Gary Macfarlane (Chief Investigator of BSRBR-AS) and the staff of the BSRBR-AS register who are currently Claudia Zabke, Elizabeth Ferguson-Jones, Maureen Heddle, Nafeesa Nazlee and Barry Morris, and to the recruiting staff at the clinical centres, details of which are available at: https://www.abdn.ac.uk/iahs/research/epidemiology/spondyloarthritis

\section{Authors' contributions}

SZ wrote the manuscript with input from all co-authors. GTJ is the Deputy Chief Investigator on BSRBR-AS and designed the study and oversaw its conduct. In the current project, he discussed results and provided input into drafts of the manuscript. KY, DMH, SJD, SKT, HL, RJM, DHS and NJG contributed towards design of the current analysis and provided input into the manuscript. All authors read and approved the final manuscript.

\section{Funding}

The BSRBR-AS is funded by the British Society for Rheumatology (BSR) who have received funding for this from Pfizer, AbbVie and UCB. These companies receive advance copies of manuscripts for comments. They have no input in determining the topics for analysis or work involved in undertaking it. SZ was supported by awards from the Royal College of Physicians (John Glyn bursary) and Royal Society of Medicine (Kovacs fellowship). KY received financial support for his doctoral study from the Pharmacoepidemiology Program at the Harvard T.H. Chan School of Public Health (partially supported by training grants from Pfizer, Takeda, Bayer and ASISA) and Honjo International Scholarship Foundation. DHS was supported by grants from the National Institute of Health (NIH-P30-AR072577 (VERITY) and NIH-K24AR055989) and has received funding from Abbvie and Amgen unrelated to this work.

\section{Availability of data and materials}

BSRBR-AS data are held at the University of Aberdeen.

\section{Ethics approval and consent to participate}

Ethical approval was obtained from the National Research Ethics Service Committee North East-County Durham and Tees Valley (reference 11/NE/ 0374) and informed consent was obtained from all participants.

\section{Consent for publication}

All authors have read and approved the manuscript for publication.

\section{Competing interests}

The authors declare that they have no competing interests.

\section{Author details}

'Musculoskeletal Biology I, Institute of Ageing and Chronic Disease, University of Liverpool, Liverpool, UK. ${ }^{2}$ Department of Academic Rheumatology, Aintree University Hospital, Liverpool, UK. ${ }^{3}$ Division of Rheumatology, Immunology and Allergy, Brigham and Women's Hospital, Boston, MA, USA. ${ }^{4}$ Departments of Epidemiology and Biostatistics, Harvard T.H. Chan School of Public Health, Boston, MA, USA. " Eppidemiology Group, School of Medicine, Medical Sciences and Nutrition, University of Aberdeen, Aberdeen, UK. ${ }^{6}$ Aberdeen Centre for Arthritis and Musculoskeletal Health, University of Aberdeen, Aberdeen, UK. ${ }^{7}$ Department of Biostatistics, Institute of Translational Medicine, University of Liverpool, Liverpool, UK. ${ }^{8}$ Department of Medicine, Harvard Medical School, Boston, MA, USA. ${ }^{9}$ Department of Orthopaedics, General Hospital of Chinese PLA, Beijing, China. ${ }^{10}$ Division of Pharmacoepidemiology and Pharmacoeconomics, Department of Medicine, Brigham and Women's Hospital and Harvard Medical School, Boston, MA, USA

\section{Received: 11 March 2019 Accepted: 8 July 2019}

Published online: 22 July 2019

\section{References}

1. Ciurea A, Scherer A, Weber U, Exer P, Bernhard J, Tamborrini G, Riek M Muller RB, Weiss B, Nissen MJ, et al. Impaired response to treatment with tumour necrosis factor alpha inhibitors in smokers with axial spondyloarthritis. Ann Rheum Dis. 2016;75(3):532-9.

2. Kydd AS, Chen JS, Makovey J, Chand V, Henderson L, Buchbinder R, Lassere M, March LM. Smoking did not modify the effects of anti-TNF treatment on health-related quality of life among Australian ankylosing spondylitis patients. Rheumatology. 2015;54(2):310-7.

3. Zhao S, Yoshida K, Jones GT, Hughes DM, Tedeschi SK, Lyu H, Moots RJ, Solomon DH, Goodson NJ. The impact of smoking on response to TNF inhibitors in axial spondyloarthritis: methodological considerations for longitudinal observational studies. Arthritis Care Res (Hoboken). 2019. https://doi.org/10.1002/acr.23851. [Epub ahead of print]

4. Glintborg B, Hojgaard P, Lund Hetland M, Steen Krogh N, Kollerup G, Jensen J, Chrysidis S, Jensen Hansen IM, Holland-Fischer M, Hojland Hansen T, et al. Impact of tobacco smoking on response to tumour necrosis factor-alpha inhibitor treatment in patients with ankylosing spondylitis: results from the Danish nationwide DANBIO registry. Rheumatology. 2016;55(4):659-68.

5. Glintborg B, Ostergaard M, Krogh NS, Dreyer L, Kristensen HL, Hetland ML. Predictors of treatment response and drug continuation in 842 patients with ankylosing spondylitis treated with anti-tumour necrosis factor: results from 8 years' surveillance in the Danish nationwide DANBIO registry. Ann Rheum Dis. 2010;69(11):2002-8.

6. Arcavi L, Benowitz NL. Cigarette smoking and infection. Arch Intern Med. 2004;164(20):2206-16

7. Pintilie M. Competing risks: a practical perspective. Chichester: Wiley; 2006

8. Macfarlane GJ, Barnish MS, Jones EA, Kay L, Keat A, Meldrum KT, Pathan E, Sturrock RD, Zabke C, McNamee P, et al. The British Society for Rheumatology Biologics Registers in Ankylosing Spondylitis (BSRBR-AS) study: protocol for a prospective cohort study of the long-term safety and quality of life outcomes of biologic treatment. BMC Musculoskelet Disord. 2015:16:347.

9. Department for Communities and Local Government. English indices of deprivation 2015 [www.gov.uk/government/statistics/english-indices-ofdeprivation-2015]. Accessed Nov 2018.

10. Hojgaard P, Glintborg B, Hetland ML, Hansen TH, Lage-Hansen PR, Petersen $\mathrm{MH}$, Holland-Fischer M, Nilsson C, Loft AG, Andersen BN, et al. Association between tobacco smoking and response to tumour necrosis factor alpha inhibitor treatment in psoriatic arthritis: results from the DANBIO registry. Ann Rheum Dis. 2015;74(12):2130-6.

11. Robins JM, Hernan MA, Brumback B. Marginal structural models and causal inference in epidemiology. Epidemiology. 2000;11(5):550-60.

12. Li F, Morgan KL, Zaslavsky AM. Balancing covariates via propensity score weighting. J Am Stat Assoc. 2018;113(521):390-400.

13. Fewell A, Hernan MA, Wolfe F, Tilling K, Choi HK, Sterne JAC. Controlling for time-dependent confounding using marginal structural models. Stata J. 2004;4(4):402-20.

14. D'Agostino RB, Lee ML, Belanger AJ, Cupples LA, Anderson K, Kannel WB. Relation of pooled logistic regression to time dependent Cox regression analysis: the Framingham Heart Study. Stat Med. 1990;9(12):1501-15.

15. Willems S, Schat A, van Noorden MS, Fiocco M. Correcting for dependent censoring in routine outcome monitoring data by applying the inverse probability censoring weighted estimator. Stat Methods Med Res. 2018; 27(2):323-35.

16. Cole SR, Hernan MA. Constructing inverse probability weights for marginal structural models. Am J Epidemiol. 2008;168(6):656-64.

17. National Institute for Health and Care Excellence. Adalimumab, etanercept and infliximab for ankylosing spondylitis [https://www.nice.org.uk/guidance/ ta143]. Accessed Nov 2018.

18. National Institute for Health and Care Excellence. TNF-alpha inhibitors for ankylosing spondylitis and non-radiographic axial spondyloarthritis [https:// www.nice.org.uk/guidance/ta383]. Accessed Nov 2018.

19. Micheroli R, Hebeisen M, Wildi LM, Exer P, Tamborrini G, Bernhard J, Moller B, Zufferey P, Nissen MJ, Scherer A, et al. Impact of obesity on the response to tumor necrosis factor inhibitors in axial spondyloarthritis. Arthritis Res Ther. 2017;19(1):164.

20. Hojgaard P, Glintborg B, Kristensen LE, Gudbjornsson B, Love TJ, Dreyer L. The influence of obesity on response to tumour necrosis factor-alpha inhibitors in psoriatic arthritis: results from the DANBIO and ICEBIO registries. Rheumatology. 2016;55(12):2191-9.

21. Chung HY, Machado P, van der Heijde D, D'Agostino MA, Dougados M. Smokers in early axial spondyloarthritis have earlier disease onset, more disease activity, inflammation and damage, and poorer function and healthrelated quality of life: results from the DESIR cohort. Ann Rheum Dis. 2012; 71(6):809-16.

22. Ciurea A, Finckh A. Smoking and spondyloarthritis. Joint Bone Spine. 2013; 80(3):234-5. 
23. Sieper J, Poddubnyy D, Pangan AL, Rathman SS, Anderson J. Smoking was not associated with response to adalimumab therapy in patients with nonradiographic axial spondyloarthritis. Ann Rheum Dis. 2013;72:s3.

24. Lord PA, Farragher TM, Lunt M, Watson KD, Symmons DP, Hyrich KL, Register BSRB. Predictors of response to anti-TNF therapy in ankylosing spondylitis: results from the British Society for Rheumatology Biologics Register. Rheumatology. 2010;49(3):563-70.

25. Dellyes A, Lafforgue P, Pradel V, Pham T: Smoking is not associated with response to TNF blockers in patients with axial spondyloarthritis. ACR/ARHP Annual Meeting 2014, Abstract no. 2584.

26. Poddubnyy D, Haibel H, Listing J, Marker-Hermann E, Zeidler H, Braun J, Sieper J, Rudwaleit M. Baseline radiographic damage, elevated acute-phase reactant levels, and cigarette smoking status predict spinal radiographic progression in early axial spondylarthritis. Arthritis Rheum. 2012;64(5):1388-98.

\section{Publisher's Note}

Springer Nature remains neutral with regard to jurisdictional claims in published maps and institutional affiliations.

Ready to submit your research? Choose BMC and benefit from:

- fast, convenient online submission

- thorough peer review by experienced researchers in your field

- rapid publication on acceptance

- support for research data, including large and complex data types

- gold Open Access which fosters wider collaboration and increased citations

- maximum visibility for your research: over $100 \mathrm{M}$ website views per year

At BMC, research is always in progress.

Learn more biomedcentral.com/submissions 